\title{
Some Generalized Results to unify Classical Polynomials
}

\author{
Mamta Dassani, Mukesh Kushwaha
}

\begin{abstract}
Present work of this paper deals with the unification of classical polynomials in which we have defined a generalized polynomial set analogous to that of associated Legendre polynomial $\mathrm{P}_{\mathrm{n}}^{\mathrm{m}}(\mathrm{X})$ by taking 5the use of Operator. Also we have derived explicit form, OperationalFormulae generating functions for this function.

Keywords: Classical Polynomials,LegendrePolynomials, Rodrigues Formula, Generating Functions.
\end{abstract}

\section{I.INTRODUCTION}

A special function is a real or complex valued function of one or more real or complex variable which is so completely that it's numerical values be tabulated. The chief organs in the study of special functions have been Rodrigue's type formula, generating functions, recurrence relations, relations with other function, operational folmulae etc. Further many various polynomials have been generalized in different directions with the help of these organs.

Classical polynomials like Legendre, Hermite, Laguerre, Jacobi, Gagenbauer and Bessel functions have been studied to a great extent and these have been generalized in a number of ways see [1]. In an attempt to unify classical polynomials of mathematical physics, Dhillon [4] considered a generalized function.

$\mathrm{z}_{\mathrm{n}}^{(\alpha, \beta, \tau)}(\mathrm{x} ; \mathrm{r}, \mathrm{s}, \mathrm{q}, \mathrm{A}, \mathrm{B}, \mathrm{m}, \mathrm{k})$ defined by the following Rodrigue's type formula:

(1.1)

$z_{n}^{(\alpha, \beta, \tau)}(x ; r, s, q, A, B, m, k)=(A x+B)^{-\alpha}\left(1-\delta x^{r}\right)^{-\beta / \tau} e^{m+n}\left[(A x+B)^{\alpha+q n}\left(1-\delta x^{r}\right)^{\beta / \tau+\delta n}\right]$ where$$
\theta \equiv \alpha^{k} D_{x}
$$$$
\mathrm{D}_{\mathrm{x}} \equiv \frac{\mathrm{d}}{\mathrm{dx}}
$$

In defining (1.1), Dhillon[4] was motivated by Rodrigue's type formula for the associated Legendre polynomials $\mathrm{P}_{\mathrm{n}}^{\mathrm{m}}(\mathrm{x})$ and the generalized function $\mathrm{P}_{\mathrm{n}}^{(\alpha, \beta, \tau)}(\mathrm{x} ; \mathrm{r}, \mathrm{s}, \mathrm{m}, \mathrm{A}, \mathrm{B})$ of Singh. A.[7] defined respectively as

Manuscript received on March20, 2021.

Revised Manuscript received on March03, 2021.

Manuscript published on March30, 2021.

* Correspondence Author

MamtaDassani*, Department of Basic Science, Bundelkhand University Jhansi(U.P), India. Email: Mamtavalecha09@gmail.com

Mukesh kushwaha, Department of Mathematical Sciences \& Computer Application, Bundelkhand University, Jhansi (U.P), India. Email: mukeshkus12785@gmail.com

(c) The Authors. Published by Blue Eyes Intelligence Engineering and Sciences Publication (BEIESP). This is an open access article under the CC BY-NC-ND license (http://creativecommons.org/licenses/by-nc-nd/4.0/)

$$
P_{n}^{m}(x)=\frac{\left(x^{2}-1\right)^{m / 2}}{2^{n} n !} \frac{d^{m+n}}{d x^{m+n}}\left(x^{2}-1\right)^{n}
$$

And

$$
P_{n}^{(\alpha, \beta, r)}(x ; r, S, m, A, B)=(A x+B)^{-\alpha}\left(1-\tau x^{r}\right)^{-\beta / \tau} D_{\alpha}^{n}\left[(A x+B)^{\alpha+m n}\left(1-\tau x^{r}\right)^{r / \tau+t+\beta n}\right]
$$

Recently in a similar manner attempt of unifying various classical polynomials of mathematical physics, Joshi and Prajapat [6] considered the operators

$$
\text { (1.5) } \mathrm{T}_{\mathrm{k}, \mathrm{q}} \equiv \mathrm{x}^{\mathrm{q}}(\mathrm{k}+\mathrm{x} \text { Dx })
$$

Where $\mathrm{k}$ is a constant and introduced the polynomial set $\left(\mathrm{M}_{\mathrm{n}}^{(\alpha)}(\mathrm{x} ; \mathrm{r} ; \mathrm{p} ; \mathrm{b} ; \mathrm{k} ; \mathrm{q}): \mathrm{n}=0,1,2 \ldots \ldots.\right)$ defined by (1.6)

$$
\left(M_{n}^{(\alpha)}(x ; r, p, b, k, q)=c(b, n) x^{-\alpha-n q-n} e^{p x^{r}} T_{k, q}^{n} \equiv\left(x^{\alpha+b n}\left(k+x e^{-p x^{r}}\right)\right.\right.
$$

Where $\mathrm{c}(\mathrm{b}, \mathrm{n})$ is a constant such that :

$$
c(b, n)=\frac{(-1)^{n / 2(b-1)(b-2)}}{2^{n b / 2(b-1)}(1)_{n b(2-b)}} b \text {, being a }
$$

non negative integer.

In view of (1.6) we introduce a generalized polynomial set $\left(S_{\mathrm{n}}^{(\alpha, \beta, \tau)}(\mathrm{x} ; \mathrm{r}, \mathrm{s}, \mathrm{q}, \mathrm{A}, \mathrm{B}, \mathrm{m}, \mathrm{k}, \mathrm{l}), \mathrm{n}=0,1,2 \ldots \ldots\right)$

defined by the following Rodrigue's formula:

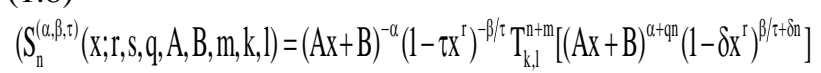

Where $\mathrm{T}_{\mathrm{k}, \mathrm{l}}^{\mathrm{n}+\mathrm{m}}=\mathrm{x}^{\mathrm{l}}(\mathrm{k}+\mathrm{X} \mathrm{Dx})$

(1.8) provides an elegant unified representation of the various known extensions of the classical polynomials and includes the polynomials due to Joshi and Singhal [10] and Chatterjea [3] as special cases and reduces to that when $\mathrm{k}=$ $0, \mathrm{l}=-1$ and $\mathrm{m}=0$

\section{II.PRELIMINARIES}

THE OPERATOR $\mathbf{T}_{k, q}$

Appell and Kampe [2] also describe some results on hypergeometric and hypershperiques function which has main results or polynomial. Gould and Hopper.[5] find some extension on the Operational formulas which are connected through with two generalization of Hermite polynomials. on the otherhand Singhal. and Savita.[8] make truthful results On a unification of generalized Humbert and Laguerre polynomials.

The operator $T_{k, q}$ has been defined as $\mathrm{T}_{\mathrm{k}, \mathrm{q}} \equiv \mathrm{x}^{\mathrm{q}}(\mathrm{k}+\mathrm{xD})$

Published By:

Blue Eyes Intelligence Engineering

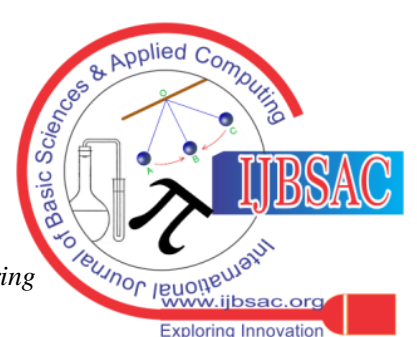




\section{Some Generalized Results to unify Classical Polynomials}

Some of the well known properties of this operator ,which we shall require in the investigations are listed below:

(2.1) $T_{\mathrm{k}, \mathrm{q}}^{\mathrm{n}}\left(\mathrm{x}^{\alpha+\mathrm{m}}\right)=\mathrm{q}^{\mathrm{n}}\left(\frac{\alpha+\mathrm{m}+\mathrm{k}}{\mathrm{q}}\right)_{\mathrm{n}} \mathrm{x}^{\alpha+\mathrm{m}+\mathrm{nq}}$

(2.2) $F\left(T_{k, q}\right)\left[x^{\alpha} f(x)\right]=x^{\alpha} F\left(T_{k, q}+x^{q} \alpha\right) f(x)$;

(2.3) $F\left(T_{k, q}\right)\left[e^{g(x)} f(x)\right]=e^{g(x)} F\left[T_{k, q}+x^{q+1} g(x)\right] f(x)$;

(2.4) $\left(T_{k, q}\right)^{n}\left[x^{j} u v\right]=x^{j} \sum_{i=0}^{n}\left({ }_{i}^{n}\right)\left(T_{k, q}^{n-i} u\right)\left(T_{q}^{i} v\right)$

(2.5) $\left(T_{k, q}\right)^{n}(u \cdot v)=\sum_{i=0}^{n}\left({ }_{i}^{n}\right)\left(T_{k, q}^{n-i} u\right)\left(T_{q}^{i} v\right)$

Where

(2.6) $\mathrm{T}_{\mathrm{q}} \equiv \mathrm{x}^{\mathrm{q}+1} \mathrm{D}_{\mathrm{x}}$;

(2.7) $\left(\mathrm{T}_{\mathrm{k}, \mathrm{q}}\right)^{\mathrm{n}}(\mathrm{xuv})=\mathrm{x} \sum_{\mathrm{i}=0}^{\mathrm{n}}\left({ }_{\mathrm{i}}^{\mathrm{n}}\right)\left(\mathrm{T}_{\mathrm{k}, \mathrm{q}}^{\mathrm{n}-\mathrm{i}} \mathrm{v}\right)\left(\mathrm{T}_{1, \mathrm{q}}^{\mathrm{i}} \mathrm{u}\right)$, Where

(2.8) $\mathrm{T}_{1, \mathrm{q}}=\mathrm{x}^{\mathrm{q}}(1+\mathrm{xD})$;

(2.9) $\quad\left(\mathrm{T}_{\mathrm{k}, \mathrm{q}}\right)^{\mathrm{n}} \mathrm{f}(\mathrm{x})=\mathrm{x}^{-\mathrm{k}} \mathrm{T}_{\mathrm{q}}^{\mathrm{n}}\left(\mathrm{x}^{\mathrm{k}} \mathrm{f}(\mathrm{x})\right.$;

$$
e^{t}\left(T_{k, q}\right)\left[x^{\alpha} f(x)\right]=\frac{x^{\alpha}}{\left(1-x^{q} q t\right)^{\alpha+k / q}} f\left[\frac{x}{\left(1-x^{q} q t\right)^{1 / q}}\right]
$$

(a);

$$
{ }_{\mathrm{T}} \mathrm{F}_{\mu}\left[\stackrel{\mathrm{T}}{\mathrm{T}} \mathrm{tT}_{\mathrm{k}, \mathrm{q}}\right] \mathrm{x}^{\alpha} \mathrm{e}^{\mathrm{px^{ \textrm {r } }}}=\sum_{\mathrm{j}=0}^{\infty} \frac{\mathrm{p}_{\mathrm{j}}}{\mathrm{j} !} \mathrm{x}^{\alpha+\mathrm{rj}}
$$$$
\text { * }
$$

${ }_{\mathrm{T}+1} \mathrm{~F}_{\mu}\left[\left(\mathrm{a}_{\mathrm{T}}\right),\left(\frac{\alpha+\mathrm{rj}+\mathrm{k}}{\mathrm{q}}\right) ;{ }_{\mathrm{x}} \mathrm{q}_{\mathrm{qt}} \mathrm{j} ;\right.$

Where $\left(a_{T}\right)$ stands for the sequence of parameters namely $a_{1}$, $\mathrm{a}_{2}, \ldots \ldots \mathrm{a}_{\mathrm{T}}$ with similar interpretation for $\left(\mathrm{b}_{\mu}\right)$.

(2.12) $\mathrm{T}_{\mathrm{q}}^{\mathrm{n}}\left[\mathrm{f}(\mathrm{z}(\mathrm{x})]=\sum_{\mathrm{i}=0}^{\mathrm{n}} \frac{(-1)^{\mathrm{i}}}{\mathrm{i} !}\left(\frac{\mathrm{d}^{\mathrm{i}}}{\mathrm{dz}}\right) \mathrm{f}(\mathrm{z}) \sum_{\mathrm{j}=0}^{\mathrm{i}}(-1)^{\mathrm{j}}\left(\mathrm{i}_{\mathrm{j}}^{\mathrm{i}}\right)(\mathrm{z}(\mathrm{x}))^{\mathrm{i}-\mathrm{j}} \mathrm{T}_{\mathrm{q}}^{\mathrm{n}}(\mathrm{z}(\mathrm{x}))^{\mathrm{j}}\right.$

\section{III.OPERATIONAL EQUATION}

Consider

$$
\begin{aligned}
& \left.\underset{\mathrm{k}, \mathrm{l}}{\mathrm{m}+\mathrm{n}}[\mathrm{Ax}+\mathrm{B})^{\alpha+\mathrm{qn}}\left(1-\tau \mathrm{X}^{\mathrm{r}}\right)^{\beta / \tau+\mathrm{sn}} \mathrm{Y}\right] \\
& =\sum_{\mathrm{i}=0}^{\mathrm{m}+\mathrm{n}}\left(\begin{array}{c}
\mathrm{m}+\mathrm{n} \\
\mathrm{i}
\end{array}\right)\left(\mathrm{T}_{\mathrm{k}, \mathrm{l}}^{\mathrm{m}+\mathrm{n}-\mathrm{i}}(\mathrm{Ax}+\mathrm{B})^{\alpha+q \mathrm{n}}\left(1-\tau \mathrm{x}^{\mathrm{r}}\right)^{\beta / \tau+\mathrm{sn}}\right)\left(\mathrm{T}_{1}^{\mathrm{i}}(\mathrm{Y})\right)
\end{aligned}
$$

(3.1) $\sum_{\mathrm{i}=0}^{m+n}\left(\begin{array}{c}m+n \\ i\end{array}\right)(A x+B)^{\alpha+q i}\left(1-\tau x^{r}\right)^{\beta / \tau /+s n} s_{n-i}^{(\alpha+q, s+s i, \tau)}(x ; r, s, A, B, m, k, l)\left(T_{1}^{i} Y\right)$

$$
\text { Using(1.8) }
$$

Where $\mathrm{Y}$ is sufficiently differentiable function of $\mathrm{x}$

Also

$$
\begin{aligned}
& \left.\mathrm{T}_{\mathrm{k}, \mathrm{l}}^{\mathrm{m}+\mathrm{n}}[\mathrm{Ax}+\mathrm{B})^{\alpha+q \mathrm{n}}\left(1-\tau \mathrm{x}^{\mathrm{r}}\right)^{\beta / \tau+\mathrm{sn}} \mathrm{Y}\right]
\end{aligned}
$$

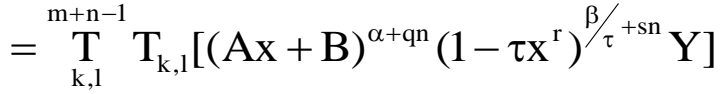

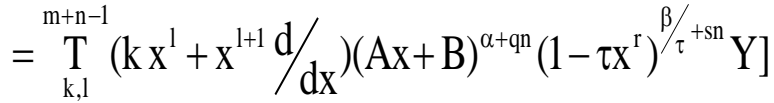

$$
\begin{aligned}
& \text { (3.2) } \left.=\mathrm{T}_{\mathrm{k}, \mathrm{l}}^{\mathrm{m}+\mathrm{n}}[\mathrm{AX}+\mathrm{B})^{\alpha+\mathrm{qn}}\left(1-\tau \mathrm{X}^{\mathrm{r}}\right)^{\beta / \tau+\mathrm{sn}} \mathrm{Y}\right]
\end{aligned}
$$

Where

$$
Y_{1}=\left(\frac{\mathrm{Ax}^{1+1}(\alpha+q n)}{(\mathrm{Ax}+\mathrm{B}}-\frac{\mathrm{rx}^{1+\mathrm{r}}(\beta+\tau s \mathrm{n})}{\left(1-\tau \mathrm{x}^{\mathrm{r}}\right.}+\mathrm{T}_{\mathrm{k}, \mathrm{l}}\right) \mathrm{Y}
$$

Similarly from equation (3.2) we see that :

$$
\left.\mathrm{T}_{\mathrm{k}, \mathrm{l}}^{\mathrm{m}+\mathrm{n}}[\mathrm{Ax}+\mathrm{B})^{\alpha+q \mathrm{n}}\left(1-\tau \mathrm{x}^{\mathrm{r}}\right)^{\beta / \tau+\mathrm{sn}} \mathrm{Y}\right]
$$$$
=\prod_{k, 1}^{m+n-2}\left[(A x+B)^{\alpha+q n}\left(1-\tau x^{r}\right)^{\beta / \tau+s n} Y_{2}\right]
$$

Where

(3.5) $Y_{2}=\left(\frac{\mathrm{Ax}^{1+1}(\alpha+q n)}{(\mathrm{Ax}+\mathrm{B}}-\frac{\mathrm{rx}^{1+\mathrm{r}}(\beta+\tau \mathrm{sn})}{\left(1-\tau \mathrm{x}^{\mathrm{r}}\right)}+\mathrm{T}_{\mathrm{k}, \mathrm{l}}\right) \mathrm{Y}_{1}$

Or using equation (3.3):

$$
Y_{2}=\left(\frac{\mathrm{Ax}^{1+1}(\alpha+q n)}{(\mathrm{Ax}+\mathrm{B}}-\frac{\mathrm{rx}^{1+\mathrm{r}}(\beta+\tau \mathrm{sn})}{\left(1-\tau \mathrm{x}^{\mathrm{r}}\right)}+\mathrm{T}_{\mathrm{k}, \mathrm{l}}\right)^{2} \mathrm{Y}
$$

Repeating the above process $(m+n-2)$ times, from (3..4)we obtain

(3.6)

$$
\begin{aligned}
& \left.\underset{\mathrm{k}, \mathrm{l}}{\mathrm{m}+\mathrm{n}}[\mathrm{Ax}+\mathrm{B})^{\alpha+q \mathrm{n}}\left(1-\tau \mathrm{x}^{\mathrm{r}}\right)^{\beta / \tau+\mathrm{sn}} Y\right] \\
& =(\mathrm{Ax}+\mathrm{B})^{\alpha+q \mathrm{n}}\left(1-\tau \mathrm{x}^{\mathrm{r}}\right)^{\beta / \tau+\mathrm{sn}}\left(\frac{\mathrm{Ax^{1+1 }}(\alpha+q n)}{(\mathrm{Ax}+\mathrm{B}}-\frac{\mathrm{rx}^{1+\mathrm{r}}(\beta+\tau \mathrm{sn})}{\left(1-\tau \mathrm{x}^{\mathrm{r}}\right)}+\mathrm{T}_{\mathrm{k}, \mathrm{l}}\right)^{\mathrm{m}+\mathrm{n}} \mathrm{Y}
\end{aligned}
$$

From equation (3.1) and (3.6) we arrive at following important operational formula:

(3.7)

$$
\begin{aligned}
& \left(\frac{\mathrm{Ax}^{1+1}(\alpha+\mathrm{qn})}{(\mathrm{Ax}+\mathrm{B}}-\frac{\mathrm{rx}^{1+\mathrm{r}}(\beta+\tau \mathrm{sn})}{\left(1-\tau \mathrm{x}^{\mathrm{r}}\right)}+\mathrm{T}_{\mathrm{k}, \mathrm{l}}\right)^{\mathrm{m}+\mathrm{n}} \mathrm{Y} \\
& =\sum_{\mathrm{i}=0}^{\mathrm{m}+\mathrm{n}}\left(\begin{array}{c}
\mathrm{m}+\mathrm{n} \\
\mathrm{i}
\end{array}\right)(\mathrm{Ax}+\mathrm{B})^{-\mathrm{q}(\mathrm{n}-\mathrm{i})}\left(1-\tau \mathrm{x}^{\mathrm{r}}\right)^{\beta / \tau}{ }^{+\mathrm{sn}} \mathrm{S}_{\mathrm{n}-\mathrm{i}}^{(\alpha+\mathrm{q}, \mathrm{s}+\tau \mathrm{s}, \tau)}(\mathrm{x} ; \mathrm{r}, \mathrm{s}, \mathrm{A}, \mathrm{B}, \mathrm{m}, \mathrm{k}, \mathrm{l})\left(\mathrm{T}_{1}^{\mathrm{i}} \mathrm{Y}\right)
\end{aligned}
$$

When $y=1$ from equation (3.7), we get

$$
\begin{aligned}
& \left(\frac{A x^{1+1}(\alpha+q n)}{(A x+B}-\frac{r x^{1+r}(\beta+\tau s n)}{\left(1-\tau x^{r}\right)}+T_{k, 1}\right)^{m+n} 1 \\
& =(A x+B)^{-q n}\left(1-\tau x^{r}\right)^{-s n} S_{n}^{\alpha, \beta, \tau}(x ; r, s, A, B, m, k, l)
\end{aligned}
$$

When $k=0, l=-1$ and $m=0$, relation (3.8) reduces to the operational formula for the function of Singh [7]:

$$
\text { (3.9) } \begin{aligned}
\left(\mathrm{D}+\frac{\mathrm{A}(\alpha+\mathrm{qn})}{(\mathrm{Ax}+\mathrm{B})}-\frac{\mathrm{rx}^{\mathrm{r}-1}-1(\beta+\tau \mathrm{sn}) \mathrm{n}}{\left(1-\tau \mathrm{x}^{\mathrm{r}}\right)}\right) \cdot 1 \\
=(\mathrm{Ax}+\mathrm{B})^{-\mathrm{qn}}\left(1-\tau \mathrm{x}^{\mathrm{r}}\right)^{-\mathrm{sn}} \mathrm{P}_{\mathrm{n}}^{\alpha, \beta, \tau}(\mathrm{x} ; \mathrm{r}, \mathrm{s}, \mathrm{q}, \mathrm{A}, \mathrm{B})
\end{aligned}
$$

Srivastava. and Singhal.[9] modified some basic polynolmial those are connected with class of polynomials defined by generalized Rodrigue's formula as similaras other formulae for the polynomials of this class can be obtained as the particular case of (3.8)

Again consider

$$
\begin{aligned}
\prod_{\mathrm{k}, \mathrm{l}}^{\mathrm{m}+\mathrm{n}}[\mathrm{A} & \left.\mathrm{B}+\mathrm{B})^{\alpha+q \mathrm{qn}}\left(1-\tau \mathrm{x}^{\mathrm{r}}\right)^{\beta / \tau+\mathrm{sn}} \mathrm{Y}\right] \\
= & \mathrm{T}_{\mathrm{k}, \mathrm{I}}^{\mathrm{m}+\mathrm{n}}\left[( \mathrm { Ax } + \mathrm { B } ) ^ { \alpha - 1 + \mathrm { qn } } ( 1 - \tau \mathrm { x } ^ { \mathrm { r } } ) ^ { \beta / \tau ^ { - 1 + \mathrm { sn } } } \left(\mathrm{kx} \mathrm{x}^{\mathrm{l}}(\mathrm{Ax}+\mathrm{B})\left(1-\tau \mathrm{x}^{\mathrm{r}}\right)\right.\right. \\
& +\mathrm{A}(\alpha+\mathrm{qn}) \mathrm{x}^{1+1}\left(1-\tau \mathrm{x}^{\mathrm{r}}\right) \mathrm{r}(\beta+\tau \mathrm{sn}) \mathrm{x}^{1+\mathrm{r}}(\mathrm{Ax}+\mathrm{B}) \\
& \left.+(\mathrm{Ax}+\mathrm{B})\left(1-\tau \mathrm{x}^{\mathrm{r}}\right) \theta \mathrm{Y}\right] ; \theta=\mathrm{x}^{1+1} \mathrm{D}
\end{aligned}
$$

Published By:

Blue Eyes Intelligence Engineering

and Sciences Publication

(C) Copyright: All rights reserved. 


$$
\begin{aligned}
& =T_{k, 1}^{m+n-2}\left[( A x + B ) ^ { \alpha + 2 + q n } ( 1 - \tau x ^ { r } ) ^ { \beta / \tau ^ { - 1 + s n } } \left(k x^{1}(A x+B)\left(1-\tau x^{r}\right)\right.\right. \\
& +A(\alpha+q n) x^{1+1}\left(1-\tau x^{r}\right)-r(\beta+\tau s n) x^{1+r}(A x+B) \\
& \left.+(A x+B)\left(1-\tau x^{r}\right) \theta\right] x\left(k x^{1}(A x+B)\left(1-\tau x^{r}\right)+A(\alpha-1+q n) x^{1+1}\left(1-\tau x^{r}\right)\right. \\
& \left.-r(\beta-\tau+\tau s n) x^{1+r}(A x+B)+(A x+B)\left(1-\tau x^{r}\right) \theta Y\right]
\end{aligned}
$$

Which by iteration gives

(3.10)

$$
\begin{aligned}
\left.\mathrm{T}_{\mathrm{k}, \mathrm{l}}^{\mathrm{m}+\mathrm{n}}[\mathrm{Ax}+\mathrm{B})^{\alpha+q \mathrm{n}}\left(1-\tau \mathrm{x}^{\mathrm{r}}\right)^{\beta / \tau+\mathrm{sn}} \mathrm{Y}\right] & \\
& =(\mathrm{Ax}+\mathrm{B})^{\alpha+(\mathrm{m}+\mathrm{n})+\mathrm{qn}}\left(1-\tau \mathrm{x}^{\mathrm{r}}\right)^{\beta / \tau-(\mathrm{m}+\mathrm{n})+\mathrm{sn}}
\end{aligned}
$$

$\prod_{i=0}^{m+n-1}\left(k x^{1}(A x+B)\left(1-\tau x^{r}\right)+A((\alpha-1+q n))^{l+1}\left(1-\tau x^{r}\right)-r(\beta-\tau+\tau s n) x^{1+r}(A x+B)+(A x+B)\left(1-\tau x^{r}\right) \theta\right] Y$

From (3.1) and (3.10) we obtain the following product type operational formula:

$\prod_{i=0}^{m+n-1}\left(k x^{l}(A x+B)\left(1-\tau x^{r}\right)+A(\alpha-i+q n) x^{l+1}\left(1-\chi^{r}\right)-r(\beta-\tau+\tau s n) x^{1+1}(A x+B)+(A x+B)\left(1-\tau x^{r}\right) x^{l+1} D\right] Y$

$=\sum_{\mathrm{i}=0}^{\mathrm{m}+\mathrm{n}}\left(\frac{\mathrm{m}+\mathrm{n}}{\mathrm{i}}\right)(\mathrm{Ax}+\mathrm{B})^{\mathrm{m}+\mathrm{n}-\mathrm{q}(\mathrm{n}-1)}\left(1-\tau \mathrm{x}^{\mathrm{r}}\right)^{\mathrm{m}+\mathrm{n}-\mathrm{s}(\mathrm{n}-\mathrm{i})}$

$* \mathrm{~S}_{\mathrm{n}-\mathrm{i}}^{(\alpha+\mathrm{i}, \beta+\tau \mathrm{si}, \tau)}(\mathrm{x} ; \mathrm{r}, \mathrm{s}, \mathrm{q}, \mathrm{A}, \mathrm{B}, \mathrm{m}, \mathrm{k}, \mathrm{l})\left(\mathrm{T}_{1}^{\mathrm{i}}(\mathrm{Y})\right)$

(3.11) gives various operational formulae for the polynomials of the class $S_{n}^{(\alpha, \beta, \tau)}(x ; r, s, q, A, B, m, k, l)$ as the special cases.

\section{IV.GENERATING FUNCTIONS:}

Using property (2.9) equation (1.8) can be written as :

$$
\begin{aligned}
& S_{n}^{(\alpha, \beta, \tau)}(x ; r, s, q, A, B, m, k, l)=(A x+B)^{-\alpha}\left(1-\tau x^{r}\right)^{\beta / \tau} \\
& { }^{*} x^{-k} e^{m+n}\left[x^{k}(A x+B)^{\alpha+q n}\left(1-\tau x^{r}\right)^{\beta / \tau+s n} ; \theta=x^{1+1} D_{x}\right. \\
& =x^{-k}(A x+B)^{-\alpha}\left(1-\tau x^{r}\right)^{\beta / \tau} \\
& *\left(\frac{\mathrm{d}}{\mathrm{du}}\right)^{\mathrm{m}+\mathrm{n}}\left[(-\mathrm{ul})^{-\mathrm{k} / \mathrm{l}}\left(\mathrm{A}(-\mathrm{ul})^{-\mathrm{i} / \mathrm{l}}+\mathrm{B}\right)^{\alpha+\mathrm{qn}}\left(1-\tau(-\mathrm{ul})^{-\mathrm{r} / \mathrm{l}}\right)^{\beta / \tau+\mathrm{sn}}\right]
\end{aligned}
$$

Where

$$
\begin{aligned}
& \mathrm{u}=-\frac{\mathrm{x}^{-1}}{\mathrm{l}} \text { gives } \\
& \frac{\mathrm{d}}{\mathrm{du}}=\mathrm{x}^{1+1} \mathrm{D}_{\mathrm{x}}
\end{aligned}
$$

The modified form of the Lagrange's expansion theorem is given by:

(4.4) $\frac{F(p)}{1-t \phi(p)}=\sum_{n=0}^{\infty} \frac{t^{n}}{n !} D^{n}\left[(\phi(x))^{n} F(x)\right]$

Where

(4.5) $\mathrm{p}=\mathrm{x}+\mathrm{t} \phi(\mathrm{p})$

And $\phi(\mathrm{p})$ is derivable at $\mathrm{p}=\mathrm{x}$ and $\phi(\mathrm{x})=0$

Using (4.3), equation (4.1) can be written as :

$\sum_{n=0}^{\infty} \frac{t^{n}}{n !} S_{n}^{(\alpha, \beta, \tau)}(x ; r, s, q, A, B, m, k, l)=x^{-k}(A x+B)^{-\alpha}\left(1-\tau x^{r}\right)^{\beta / \tau}$

$* \sum_{\mathrm{n}=0}^{\infty} \frac{\mathrm{t}^{\mathrm{n}}}{\mathrm{n} !}\left(\mathrm{x}^{\mathrm{l}+1} \mathrm{D}_{\mathrm{x}}\right)^{\mathrm{m}+\mathrm{n}}\left[\left\{\left(\mathrm{A}(-\mathrm{ul})^{-\mathrm{i} / 1}+\mathrm{B}\right)^{\mathrm{q}}\left(1-\tau(-\mathrm{ul})^{-\mathrm{r} / 1}\right)^{\mathrm{s}}\right\}^{\mathrm{n}}\right]$

$$
*\left[(-\mathrm{ul})^{-\mathrm{k} / \mathrm{l}}\left(\mathrm{A}(-\mathrm{ul})^{-\mathrm{i} / \mathrm{l}}+\mathrm{B}\right)^{\alpha}\left(1-\tau(-\mathrm{ul})^{-\mathrm{r} / \mathrm{l}}\right)^{\beta / \tau}\right]
$$

Or

(4.6) $\sum_{\mathrm{n}=0}^{\infty} \frac{\mathrm{t}^{\mathrm{n}}}{\mathrm{n} !} S_{\mathrm{n}}^{(\alpha, \beta, \tau)}(\mathrm{x} ; \mathrm{r}, \mathrm{s}, \mathrm{q}, \mathrm{A}, \mathrm{B}, \mathrm{m}, \mathrm{k}, \mathrm{l}) * \mathrm{x}^{\mathrm{k}}(\mathrm{Ax}+\mathrm{B})^{\alpha}\left(1-\tau \mathrm{x}^{\mathrm{r}}\right)^{\mathrm{\beta} / \tau}$

$$
=\left(x^{l+1} D_{x}\right)^{m} * \sum_{n=0}^{\infty} \frac{t^{n}}{n !}\left(x^{1+1} D\right)\left[(\phi(u))^{n} \cdot F(u)\right]
$$

Where

(4.7)

$$
\phi(\mathrm{u})=\left[\left(\mathrm{A}(-\mathrm{ul})^{-\mathrm{i} / \mathrm{l}}+\mathrm{B}\right)^{\mathrm{q}}\left(1-\tau(-\mathrm{ul})^{-\mathrm{r} / \mathrm{l}}\right)^{\mathrm{s}}\right]
$$

And

(4.8) $\mathrm{F}(\mathrm{u})=\left[(-\mathrm{ul})^{-\mathrm{k} / \mathrm{l}}\left(\mathrm{A}(-\mathrm{ul})^{-\mathrm{i} / \mathrm{l}}+\mathrm{B}\right)^{\alpha}\left(1-\tau(-\mathrm{ul})^{-\mathrm{r} / \mathrm{l}}\right)^{\mathrm{s}}\right]$

Now applying Lagrange's theorem(4.4)on R.H.S of equation (4.6) and by use of the equation (4.7) and (4.8) we obtain the required generating relation as:

$$
\begin{gathered}
x^{\mathrm{k}}(\mathrm{Ax}+\mathrm{B})^{-\alpha}\left(1-\tau \mathrm{x}^{\mathrm{r}}\right)^{\beta / \tau} \sum_{\mathrm{n}=0}^{\infty}\left(\mathrm{S}_{\mathrm{n}}^{(\alpha, \beta, \tau)}(\mathrm{x} ; \mathrm{r}, \mathrm{s}, \mathrm{q}, \mathrm{A}, \mathrm{B}, \mathrm{m}, \mathrm{k}, \mathrm{l}) \frac{\mathrm{t}^{\mathrm{n}}}{\mathrm{n} !}\right. \\
=\left(\mathrm{x}^{\mathrm{l}+1} \mathrm{D}_{\mathrm{x}}\right)^{\mathrm{m}} \frac{\left[(-\mathrm{pl})^{-\mathrm{k} / \mathrm{l}}\left(\mathrm{A}(-\mathrm{pl})^{-\mathrm{i} / 1}+\mathrm{B}\right)^{\alpha}\left(1-\tau(-\mathrm{pl})^{-\mathrm{r} / \mathrm{l} /)^{\beta / \mathrm{r}}}\right]\right.}{1+\tau \mathrm{rst}(-\mathrm{pl})^{(-\mathrm{r}+1) / \mathrm{l}}\left(\mathrm{A}(-\mathrm{pl})^{-1 / 1}+\mathrm{B}\right)^{\alpha}\left(1-\tau(-\mathrm{pl})^{-\mathrm{r} / 1}\right)^{\mathrm{s}-1}-\mathrm{Aqt}(-\mathrm{pl})^{-(1+1) / 1}} \\
* \frac{1}{\left(\mathrm{~A}(-\mathrm{pl})^{-\mathrm{i} / 1}+\mathrm{B}\right)^{\mathrm{q}-1}\left(1-\tau(-\mathrm{pl})^{-\mathrm{r} / 1}\right)^{\mathrm{s}}}
\end{gathered}
$$

Where

$$
\mathrm{p}=\mathrm{u}+\mathrm{t}\left(\mathrm{A}(-\mathrm{pl})^{-\mathrm{i} / \mathrm{l}}+\mathrm{B}\right)^{\mathrm{q}}\left(1-\tau(-\mathrm{pl})^{-\mathrm{r} / \mathrm{l}}\right)^{\mathrm{s}}
$$

Or

(4.9) $x^{-k}(A x+B)^{-\alpha}\left(1-\tau x^{r}\right)^{\beta / \tau}$

$$
\begin{aligned}
& *\left(\mathrm{x}^{\mathrm{l}+1} \mathrm{D}_{\alpha}\right)^{\mathrm{m}}\left[\frac{(-\mathrm{pl})^{-\mathrm{k} / \mathrm{l}} \mathrm{x}^{\alpha} \mathrm{y}^{\beta / \tau}}{1+\tau \mathrm{rst}(-\mathrm{pl})^{(-\mathrm{r}+1) / 1} \mathrm{x}^{\mathrm{q}} \mathrm{y}^{\mathrm{s}-1}-\mathrm{Aqt}(-\mathrm{pl})^{-(1+1) / 1} \mathrm{x}^{\mathrm{q}-1} \mathrm{y}^{\mathrm{s}}}\right] \\
& =\sum_{\mathrm{n}=0}^{\infty} \frac{\mathrm{t}^{\mathrm{n}}}{\mathrm{n} !} \mathrm{S}_{\mathrm{n}}^{(\alpha, \beta, \tau)}(\mathrm{x} ; \mathrm{r}, \mathrm{s}, \mathrm{q}, \mathrm{A}, \mathrm{B}, \mathrm{m}, \mathrm{k}, \mathrm{l})
\end{aligned}
$$

Where,

(4.10) $\quad \mathrm{p}=\mathrm{u}+\mathrm{x}^{\mathrm{q}} \mathrm{y}^{\mathrm{s}} \mathrm{t}$;

$$
\mathrm{X}=\left(\mathrm{A}(-\mathrm{pl})^{-\mathrm{i} / \mathrm{l}}+\mathrm{B}\right)
$$

And

$$
y=\left(1-\tau(-p l)^{-r / l}\right)
$$

\section{V.GENERALISED RESULTS}

The generating relation (4.9)-(4.12) is a generalization of so many well known generating relations .For example taking $\alpha=0, \beta=0, \tau=1, r=1, s=1, q=1, A=1, B=1, k=0,1=-1 \quad$ from equation (4.9) and (4.10) we get;

$$
\mathrm{D}^{\mathrm{m}}\left[\frac{1}{1+2 \mathrm{pt}}\right]=\sum_{\mathrm{n}=0}^{\infty} \mathrm{S}_{\mathrm{n}}^{(0,0,1)}(\mathrm{x} ; 1,1,1,1,1, \mathrm{~m}, 0,-1) \frac{\mathrm{t}^{\mathrm{n}}}{\mathrm{n} !}
$$

Where

(5.2) $\mathrm{p}=\mathrm{x}+\mathrm{t}\left(1-\mathrm{p}^{2}\right)$

Equation (5.2) on solving for $\mathrm{p}$ gives

(5.3) $1+2 \mathrm{pt}=\sqrt{\left(4 \mathrm{t}^{2}+4 \mathrm{tx}+1\right)}$

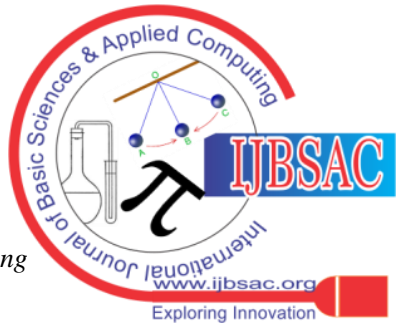




\section{Some Generalized Results to unify Classical Polynomials}

Using (5.3) and (4.12) equation (5.1) takes the form:

$\left.D^{m}\left(4 t^{2}+4 t x+1\right)^{-1 / 2}\right]=\sum_{n=0}^{\infty}(-2)^{n} n !(x-1)^{-m / 2} P_{n}^{m}(x) \frac{t^{n}}{n !}$

Or

$\left(x^{2}-1\right)^{m / 2} D^{m}\left[\left(1+w^{2}+2 w x\right)^{-1 / 2}\right]=\sum_{n=0}^{\infty} P_{n}^{m}(x)(-w)^{n}$

Or,

$(5,4)$

$\left(\mathrm{x}^{2}-1\right)^{\mathrm{m} / 2}(-1)^{\mathrm{m}}(/ 2)_{\mathrm{m}}(2 \mathrm{w})^{\mathrm{m}}\left(1+\mathrm{w}^{2}+2 \mathrm{wx}\right)^{-1 / 2-\mathrm{m}}=\sum_{\mathrm{n}=0}^{\infty} \mathrm{P}_{\mathrm{n}}^{\mathrm{m}}(\mathrm{x})(-\mathrm{w})^{\mathrm{n}}$

Where $2 \mathrm{w}=\mathrm{t}$

(5.4) is the generating relation for associated Legendre function (5.1).

Similarly taking

$\alpha=\mathrm{a}, \beta=\mathrm{p}, \tau=0, \mathrm{~s}=0, \mathrm{q}=0, \mathrm{~A}=1, \mathrm{~B}=0, \mathrm{k}=0, \mathrm{l}=-1$

From equation (4.9) we obtain the following generating relation for the function of Gould and Hopper[5];

$(p / x)^{a} \exp \left[v^{r}\left(1-(1+t / x)^{r}\right)\right]=\sum_{n=0}^{\infty} H_{n}^{r}(x, a, p) \frac{t^{n}}{n !}$

We can write

$$
\begin{aligned}
& e^{t s} f(x)=\sum_{n=0}^{\infty} \frac{t^{n}}{n !} S^{n} f(x) \\
& =\sum_{n=0}^{\infty} \frac{t^{n}}{n !} \sum_{i=0}^{n}\left(\begin{array}{l}
n \\
i
\end{array}\right)(A x+B)^{-q(n-i)}\left(1-\tau x^{r}\right)^{-s(n-i)} \\
& =\sum_{n=0}^{\infty} \frac{t^{n}}{n !}(A x+B)^{-q n}\left(1-\tau x^{r}\right)^{-s n} S_{n}^{(\alpha-q n, \beta-\tau n, \tau)}(x ; r, s, q, A, B, m, k, l) \\
& * \sum_{i=0}^{\infty} \frac{t^{i}}{i !} T_{1}^{i} f(x)
\end{aligned}
$$

[Using(1.8)]

$=(A x+B)^{-\alpha}\left(1-\tau x^{r}\right)^{-\beta / \tau}\left[T_{k, l}^{m}\left\{e^{t} T_{k, l}(A x+B)^{\alpha}\left(1-\tau x^{r}\right)^{\beta / \tau}\right\}\right]$

$$
*^{\mathrm{t}} \mathrm{T}_{\mathrm{l}} \mathrm{f}(\mathrm{x})
$$

$=(A x+B)^{-\alpha}\left(1-\tau x^{r}\right)^{-\beta / \tau} T_{k, l}^{m}\left[\left(1-1 x^{1} t\right)^{-k / l}\left(A x\left(1-x^{1} l t\right)^{-1 / l}+B\right)^{\alpha}\right.$

$\left.*\left(1-\tau \mathrm{x}\left(1-\mathrm{x}^{1} \mathrm{lt}\right)^{-\mathrm{r} / \mathrm{l}}\right)^{\beta / \tau}\right] * \mathrm{f}\left(\mathrm{x}\left(1-\mathrm{x}^{\mathrm{l}} \mathrm{lt}\right)^{-1 / \mathrm{l}}\right]$

$=(A X+B)^{-\alpha}\left(1-\tau X^{r}\right)^{-\beta / \tau-k}\left(X^{l+1} D_{x}\right)^{m}\left[X^{k}\left(1-X^{1} l t\right)^{-k / l}\right]$

$\left.*\left(A x\left(1-X^{l} l t\right)^{-1 / l}+B\right)^{\alpha}\left(1-\tau X^{r}\left(1-X^{1} l t\right)^{-r / l}\right)^{\beta / \tau}\right] * f\left(X(1-X l l t)^{-1 / l}\right]$

$=X^{-k}(A x+B)^{-\alpha}\left(1-\tau x^{r}\right)^{-\beta / \tau}\left(X^{1+1} D_{x}\right)^{m}[F(x)] f\left(x\left(1-x^{1} 1 t\right)^{-1 / 1}\right]$

Where

$$
F(x)=x^{k} c^{k}(A x c+B)^{\alpha}\left(1-\tau c^{r} x^{r}\right)^{\beta / \tau}
$$

And

$$
\mathrm{C}=\left(1-\mathrm{x}^{\mathrm{l}} \mathrm{lt}\right)^{-1 / \mathrm{l}}
$$

To find the value of $\left(x^{1+1} D_{x}\right)[F(x)]$ in $(5,7)$ we use the following formula given by Srivastava and Singhal.[9]
$\mathrm{D}_{\mathrm{g}(\mathrm{x})}^{\mathrm{m}}\{\mathrm{F}(\mathrm{x})\}=\mathrm{D}_{\mathrm{w}}^{\mathrm{m}}\{\mathrm{F}(\mathrm{x})\} \mathrm{g}^{\mathrm{l}}(\mathrm{x})\left(\left(\frac{\mathrm{x}-\mathrm{v}}{\mathrm{g}(\mathrm{x})-\mathrm{g}(\mathrm{v})}\right)^{\mathrm{m}+1}\right)_{\mathrm{v}=\mathrm{x}}$

Where

$$
D_{g(x)}=\frac{d}{\operatorname{dg}(x)}
$$

Hence

$$
\mathrm{x}^{1+1} \mathrm{D}_{\mathrm{x}}=\frac{1}{\mathrm{x}^{-1-1}}\left(\frac{\mathrm{d}}{\mathrm{dx}}\right)=\frac{\mathrm{d}}{\mathrm{d}\left(-\frac{\mathrm{x}^{-1}}{1}\right)}
$$

Hence

$$
g(x)=-\frac{1}{1} x^{-1}
$$

Therefore from $(5,11)$ :

$\left(x^{l+1} D_{x}\right)^{m}[F(x)]=D_{w}^{m}\left(x^{k} c^{k}(A x c+B)^{\alpha}\left(1-\tau c^{r} x^{r}\right)^{\beta / \tau} x^{-l-1}\left(\frac{x-v}{\frac{-1}{l} x^{-1}+\frac{1}{l} v^{-1}}\right)^{m+n}\right.$ $=D_{w}^{m}\left(x^{k-1-1} c^{k}(A x c+B)^{\alpha}\left(1-\tau c^{r} x^{r}\right)^{\beta / \tau} l^{m+1}\left(\frac{u}{x^{-1}-(u+x)^{-1}}\right)^{m+1}\right)_{u=0}$

[On substituting $\mathrm{v}=\mathrm{u}+\mathrm{x}$ ]

$=D_{\mathrm{w}}^{\mathrm{m}}\left(\mathrm{l}^{\mathrm{m}+1} \mathrm{X}^{\mathrm{k}-1-1} \mathrm{c}^{\mathrm{k}}(\mathrm{Axc}+\mathrm{B})^{\alpha}\left(1-\tau \mathrm{c}^{\mathrm{r}} \mathrm{x}^{\mathrm{r}}\right)^{\beta / \tau} \mathrm{l}\left(\frac{\mathrm{ux}}{1-(1+\mathrm{u} / \mathrm{x})^{-1}}\right)^{\mathrm{m}+1}\right)_{\mathrm{u}=0}$

With the help of (5.14), from equation (5.7) we get:

$e^{t S} f(x)=x^{-k}(A x+B)^{-\alpha}\left(1-\tau x^{r}\right)^{-\beta / \tau}$

$=\left[D_{\mathrm{w}}^{\mathrm{m}}\left(1^{\mathrm{m}+1} \mathrm{x}^{\mathrm{k}-1-1} \mathrm{c}^{\mathrm{k}}(\mathrm{Axc}+\mathrm{B})^{\alpha}\left(1-\tau \mathrm{c}^{\mathrm{r}} \mathrm{x}^{\mathrm{r}}\right)^{\beta / \tau} \mathrm{l}\left(\frac{\mathrm{ux} \mathrm{x}^{1}}{1-(1+\mathrm{u} / \mathrm{x})^{-1}}\right)^{\mathrm{m}+1}\right)_{\mathrm{u}=0}\right] \mathrm{xf}(\mathrm{xc})$

Or,

$e^{t s} f(x)=x^{-k}(A x+B)^{-\alpha}\left(1-\tau x^{r}\right)^{-\beta / \tau}\left[D_{w}^{m} \psi(x, w, u)\right]_{u=0} f(x c)$

Where

(5.16)

$(x, u, w)=l^{m+1} x^{k-1-1} c^{k}(A x c+B)^{\alpha}\left(1-\tau c^{r} x^{r}\right)^{\beta / \tau} l\left(\frac{u x^{1}}{1-(1+u / x)^{-1}}\right)^{m+1}$

Taking

$$
f(x)=S_{n}^{(\alpha, \beta, \tau)}(x ; r, s, q, A, B, m, k, l)
$$

From equation (5.5) we get :

$$
\begin{aligned}
& \sum_{j=0}^{\infty} \frac{t^{j}}{j !} S^{j} S_{n}^{(\alpha, \beta, \tau)}(x ; r, s, q, A, B, m, k, l) \\
& =(A x+B)^{-\alpha}\left(1-\tau x^{r}\right)^{-\beta / \tau} T_{k, l}^{m}\left[\left(1-1 x^{1} t\right)^{-k / l}\left(A x\left(1-x^{l} l t\right)^{-1 / l}+B\right)^{\alpha}\right. \\
& \left.\left.\quad\left(1-\tau x^{r}\right)\left(1-x^{1} l t\right)^{-r / l}\right)^{\beta / \tau}\right] \\
& * S_{n}^{(\alpha, \beta, \tau)}\left(x\left(1-x^{1} l t\right)^{-l / 1} ; r, s, q, A, B, m, k, l\right)(5.17)
\end{aligned}
$$




$$
\begin{aligned}
& \sum_{j=0}^{\infty} \frac{\mathrm{t}^{\mathrm{j}}}{\mathrm{j} !}(\mathrm{Ax}+\mathrm{B})^{-\mathrm{qj}}\left(1-\tau \mathrm{x}^{\mathrm{r}}\right)^{-\mathrm{sj}} S_{\mathrm{n}+\mathrm{j}}^{(\alpha-\mathrm{q} ; \beta-\tau \mathrm{s}, \tau)}(\mathrm{x} ; \mathrm{r}, \mathrm{s}, \mathrm{q}, \mathrm{A}, \mathrm{B}, \mathrm{m}, \mathrm{k}, \mathrm{l}) \\
& =(A x+B)^{-\alpha}\left(1-\tau x^{r}\right)^{-\beta / \tau} T_{k, l}^{m}\left[\left(1-1 x^{1} t\right)^{-k / l}\left(A x\left(1-x^{l} l t\right)^{-1 / l}+B\right)^{\alpha}\right. \\
& \left.\left.*\left(1-\tau \mathrm{X}^{\mathrm{r}}\right)\left(1-\mathrm{X}^{1} \mathrm{lt}\right)^{-\mathrm{r} / \mathrm{l}}\right)^{\beta / \tau}\right] \\
& * S_{\mathrm{n}}^{(\alpha, \beta, \tau)}\left(\mathrm{x}\left(1-\mathrm{x}^{1} \mathrm{lt}\right)^{-\mathrm{l} / 1} ; \mathrm{r}, \mathrm{s}, \mathrm{q}, \mathrm{A}, \mathrm{B}, \mathrm{m}, \mathrm{k}, \mathrm{l}\right)
\end{aligned}
$$

(5.17) gives another generating function for the polynomial set in particular for associated Legendre function Now(5.17) reduces to:

$$
\begin{gathered}
\sum_{j=0}^{\infty} \frac{t^{j}}{j !}(x+i)^{j}(1-x)^{j}(-2)^{n+j}(n+j) !\left(x^{2}-1\right)^{-m / 2} P_{n+j}^{m}(x) \\
=D^{m}\left[(-2)^{m} n !((x+t)-1)^{-m / 2} P_{n}^{m}(x+t)\right]
\end{gathered}
$$

Or

$$
\begin{aligned}
& \text { (5.18) } \sum_{j=0}^{\infty}\left(\frac{n+j}{j}\right) w^{j} P_{n+j}^{m}(x) \\
& \left.=\left(x^{2}-1\right)^{-m / 2} D^{m}\left[\left((x+w)\left(1-x^{2}\right)\right)^{2}-1\right)^{-m / 2} P_{n}^{m}\left(x+w\left(1-x^{2}\right)\right)\right]
\end{aligned}
$$

Where

$$
\frac{\mathrm{t}}{\left(1-\mathrm{x}^{2}\right)}=\mathrm{w}
$$

Relations (5.6) and (5.17) are to bethe new generating relations.

\section{VI.RESULT}

The further generalized result is given by equation (5.6) which is shown as:

$$
\begin{aligned}
& \sum_{j=0}^{\infty} \frac{t^{j}}{j !}(A x+B)^{-q j}\left(1-\tau x^{r}\right)^{-s j} S_{n+j}^{(\alpha-q j ; \beta-\tau s j, \tau)}(x ; r, s, q, A, B, m, k, l) \\
& =(A x+B)^{-\alpha}\left(1-\tau x^{r}\right)^{-\beta / \tau} T_{k, l}^{m}\left[\left(1-1 x^{1} t\right)^{-k / l}\left(A x\left(1-x^{l} l t\right)^{-1 / l}+B\right)^{\alpha}\right. \\
& \left.*\left(1-\tau x\left(1-x^{1} l t\right)^{-r / l}\right)^{\beta / \tau}\right]^{*} f\left(x\left(1-x^{l} l t\right)^{-1 / l}\right] \\
& \sum_{j=0}^{\infty} \frac{t^{j}}{j !}(A x+B)^{-q j}\left(1-\tau x^{r}\right)^{-s j} S_{n+j}^{(\alpha-q j ; \beta-\tau s j, \tau)}(x ; r, s, q, A, B, m, k, l) \\
& =(A x+B)^{-\alpha}\left(1-\tau x^{r}\right)^{-\beta / \tau} T_{k, l}^{m}\left[\left(1-1 x^{1} t\right)^{-k / l}\left(A x\left(1-x^{l} l t\right)^{-1 / l}+B\right)^{\alpha}\right. \\
& \left.\left.\left(1-\tau x^{r}\right)\left(1-x^{1} l t\right)^{-r / l}\right)^{\beta / \tau}\right] \\
& * S_{n}^{(\alpha, \beta, \tau)}\left(x\left(1-x^{1} l t\right)^{-l / 1} ; r, s, q, A, B, m, k, l\right)
\end{aligned}
$$

\section{VII.CONCLUSION}

The present paper deals with the unification of classical polynomial in which we have define generalized polynomial set analogous to rate of associated Legendre polynomials. In which we have drive explicit form, operational formula and generating function for this function.

\section{REFERENCES}

1. R. Appell., Suriune suite de polynomials ayanttoutesleuresrecinescellesArchivder Math. Uni associated orthogonal polynomials, 1901, pp. 1-44. pp. 69

2. P. Appell. and Kampe-de-Feriet., Function hypergeometric and hypershperiques: polynomials Hermite, Gauthier Villars Paris., 1926.
3. S.K. Chaterjee., Some operational formula connected with a function defined by a generalized Rodrigue's formula, Acta. Math. ,1966, pp 379-385.

4. S.S. Dhillon., A study of generalization of Special functions of mathematical physics and their applications, Ph.D. thesis B.U. Univ. Jhansi., 1989.

5. H.W. Gould and A.T. Hopper., Operational formulas connected with two generalization of Hermite polynomials Duke Math. Jour. 29 1962,pp 51-64.

6. 6. C.M. Joshi and M.L. Prajapati., The operator an a generalization of certain classical polynomials; Kyungpook Math. Jour. 15, 1975, pp 191-199

7. A. Singh., A study of special function of Mathematical physics and their applications in combinational analyhsis, Ph.D. thesis, B. Univ. Jhansi. ,1981.

8. J.P. Singhal., and K. Savita., On a unification of generalized Humbert and Laguerre polynomials, Jnanabha, Vol. 9/10, 1980, pp. 171-178.

9. H.M. Srivastava. and J.P. Singhal., A class of polynomials defined by generalized Rodrigue's formula : Ann. Mat. Pura. App. (14), 40 an 90, 1971, pp. 75-85 and pp. 345-353.

10. C.M. Joshi. and J.P. Singhal., Operational formulae associated with a class of polynomials unifying the generalized Laguerre and Hermite polynomials, Riv. Mat. Univ. Pharma (3), 1, 1972, pp. 279-286.

\section{AUTHORS PROFILE}

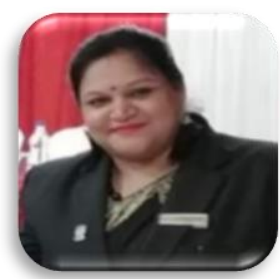

Mamata Dassanihas, 15 years teaching experience and presently working as teaching assistant in the Department of Basic Science, Bundelkhand University, Jhansi, India. She received her Ph.D degree from Barkatullah University, Bhopal(M.P). she has presented many research papers in international meets. his research area is functional analysis.

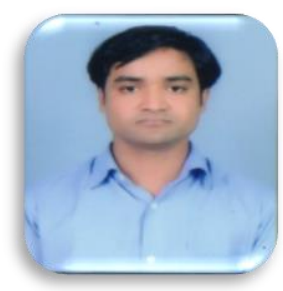

Mukesh Kushwaha, has 7 years of teaching experience and presently working as teaching assistant in the Department of Basic Science, Bundelkhand University, Jhnasi, India. He has published many research papers in reputed journal and presented many research papers in international meets. his research interests are Fixed Point Theory, Fuzzy Optimization, Neural Network.
Published By:

Blue Eyes Intelligence Engineering and Sciences Publication

(C) Copyright: All rights reserved.

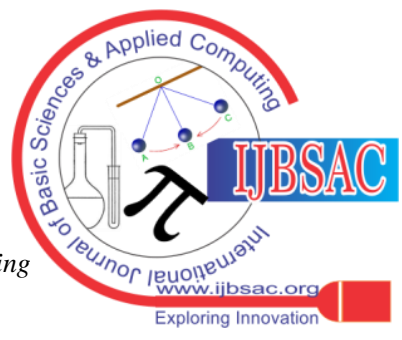

